\title{
Testing of mechanisms of action of rituximab and clinical results in high-risk patients with aggressive CD20+ lymphoma
}

\section{Barbara Jezeršek Novaković ${ }^{1}$, Vladimir Kotnik ${ }^{2}$, Tanja Južnič Šetina ${ }^{1}$, Marjeta Vovk ${ }^{1}$, Srdjan Novakovic ${ }^{3}$}

${ }^{1}$ Department of Medical Oncology, Institute of Oncology Ljubljana; ${ }^{2}$ Institute of Microbiology and Immunology, Medical Faculty Ljubljana; Department of Molecular Diagnostics,

Institute of Oncology Ljubljana, Ljubljana, Slovenia

\begin{abstract}
Background. Rituximab has been applied successfully in the treatment of indolent and aggressive CD20 positive B cell lymphomas, yet the exact in vivo mechanisms of its action have not been unambiguously explained. This study was therefore aimed to confirm the presumed major mechanisms of action of rituximab and concomitantly to assess the effectiveness of first-line chemoimmunotherapy in high-risk patients with aggressive CD20 lymphomas.

Patients, materials and methods. The activity of rituximab was tested in vitro on Raji and SU-DHL-4 cells using the cell proliferation assay and flow cytometry. In the clinical part of the study, 20 high-risk patients with aggressive CD 20 lymphomas were treated with $\mathrm{R}$-CHOP.

Results. Only complement-mediated cytotoxicity was observed under the in vitro applied experimental conditions. Neither the direct apoptotic effect nor the antibody-dependent cell-mediated cytotoxicity was detected probably due to a too low concentration of rituximab and a too low ratio of cytotoxic lymphocytes to tumor cells. The treatment outcome in patients was excellent since complete remissions were achieved in $90 \%$ of poor-risk patients at the end of primary treatment and $80 \%$ of patients were disease-free at 18.5 months median observation period.

Conclusions. According to our results, the complement-dependent cytotoxicity is an important mechanism of rituximab action in vitro. To achieve direct apoptosis, higher concentrations than $20 \mu \mathrm{g} / \mathrm{ml}$ of rituximab should be used, while for an effective antibody-dependent cell-mediated cytotoxicity, the ratio of cytotoxic lymphocytes to tumor cells should be higher than 1:1. In the high-risk patients with aggressive CD20 lymphomas, the addition of rituximab to CHOP substantially improves the therapeutic results.
\end{abstract}

Key words: lymphoma, B-cell - therapy; antigens, CD 20; antibodies, monoclonal

Received 5 February 2007

Accepted 9 March 2007

Correspondence to: Dr. Srdjan Novaković, D.Sc. Institute of Oncology Ljubljana, Zaloška 2, 1000 Ljubljana, Slovenia. Tel.: +386 15879 432; Fax: +386 1 5879 434; E-mail: snovakovic@onko-i.si

\section{Introduction}

The CD20 antigen, a $35 \mathrm{kDa}$ phosphoprotein, is restricted to the $\mathrm{B}$ cell lineage and is expressed by mature B cells and most malignant B cell lymphomas. While 
the exact functions of CD20 are presently unknown, it is considered to be involved in many cellular signaling events, including proliferation, activation, differentiation, and apoptosis upon crosslinking. Its attributes, as its tetraspan binding in the cell membrane and the lack of internalization or downregulation upon antibody binding, make CD20 a suitable target for an effective antibody. 1,2

Rituximab, a chimeric (human-mouse) IgG $\kappa$ monoclonal antibody that recognizes the CD20 antigen ${ }^{3}$, is the most widely recognized and used monoclonal antibody in the B cell lymphoid malignancies. It has been applied successfully in the treatment of indolent CD20 positive B cell lymphomas and, more recently, also in the treatment of aggressive lymphomas in combination with standard chemotherapy. The indications for its use are now expanding to autoimmune diseases. ${ }^{4}$

The mechanisms of anti-tumor action of rituximab are diverse. The human IgG component of the antibody is able to bind human complement (CDC) and also interact with effector cells to kill cells by antibody-dependent cell-mediated cytotoxicity (ADCC). Some investigators have also shown direct effects of the antibody on human tumor cell lines expressing CD20. These effects include inhibition of proliferation, induction of apoptosis and increased sensitivity to chemotherapeutic agents, although the extent to which each of these mechanisms may contribute to the anti-tumor action of rituximab remains to be determined. Rituximab thus acts by additional mechanisms compared to conventional chemotherapeutic agents. The chimeric nature of the antibody results in minimal immunogenicity and allows repeated use. The antibody may be combined with conventional chemotherapy, with potential for increased efficacy and minimal added toxicity. ${ }^{5}$
Due to still unambiguously explained mechanisms of action, we aimed our study at additional in vitro testing of rituximab activities. There were predominately three questions we wanted to resolve - firstly, to confirm the in vitro anti-tumor activity of the antibody against established CD20 positive lymphoma cell lines, secondly, to evaluate the importance of the three major mechanisms of action (i.e. complement mediated cytotoxicity, antibody mediated cellular cytotoxicity and apoptosis) in in vitro conditions, and thirdly, to determine some of the pharmacokinetic data (the time needed for rituximab binding as well as the duration of rituximab-CD20 binding). Furthermore, a smaller clinical research was performed in order to determine the effectiveness of first-line chemoimmunotherapy with rituximab and CHOP (R-CHOP) in the high-risk patients with aggressive CD20 positive NonHodgkin's lymphoma (NHL).

\section{Patients, materials and methods}

\section{Cell cultures}

The CD20+ human B-cell lines - Raji (American Type Culture Collection - ATCC, Rockville, MD, USA) and SU-DHL-4 (Deutsche Sammlung von Mikroorganismen und Zellkulturen - DSMZ, Braunschweig, D) were grown in RPMI 1640, 25 mM HEPES (Gibco, Invitrogen, Grand Island, NY) supplemented with $10 \%$ of inactivated fetal calf serum - iFCS (Sigma, St. Louis, MO), penicillin (100 units/ml, Pliva, Zagreb, CRO), and gentamycin $(50 \mu \mathrm{g} / \mathrm{ml}, \mathrm{Krka}$, Novo mesto, SLO).

Isolation of human monocytes from peripheral blood ( $h P B M C)$

Human PBMC were isolated on FicollPaque (Amersham Pharmacia Biotech AB, Uppsala, S). 
Determination of mechanisms of action of rituximab - design of in vitro experiments

In the experiments, CD20+ human B-cell lines Raji and SU-DHL-4 were used. When the cells in the tissue culture reached the optimal density ( $70-80 \%$ of confluence), the growth medium was removed and replaced with the growth medium (RPMI) containing the following tested components:

$\cdot 2 \%$ of inactivated fetal calf serum - FCS,

- $2 \%$ of inactivated human serum - iHS,

- $20 \%$ intact human serum - HS,

- $2 \%$ FCS + fresh hPBMC (ratio of target cells and lymphocytes - 1:1),

- $2 \% \mathrm{FCS}+20 \mu \mathrm{g} / \mathrm{ml}$ of rituximab,

- $2 \% \mathrm{iHS}+20 \mu \mathrm{g} / \mathrm{ml}$ of rituximab,

- $20 \% \mathrm{HS}+20 \mu \mathrm{g} / \mathrm{ml}$ of rituximab,

- $2 \%$ FCS + fresh hPBMC (ratio of target cells and lymphocytes - 1:1) $+20 \mu \mathrm{g} / \mathrm{ml}$ of rituximab.

The samples were taken from the culture 4 hours, 24 hours and 48 hours after the beginning of treatment.

The saturation of the CD20 epitopes on lymphocytes with rituximab was determined at each point of the experiment.

\section{Cell proliferation assay}

Biological test used for the detection of cell proliferation was the CellTiter $96^{\circledR} \mathrm{A}_{\text {queous }}$ Non-Radioactive Cell Proliferation Assay (Promega, Madison, WI, USA). This is a colorimetric method for determining the number of viable cells in chemosensitivity assays. The method is based on the detection of MTS (tetrazolium compound) bioreduction into a formazan product that is soluble in tissue culture medium. The absorbance of the formazan was measured at $490 \mathrm{~nm}$. In the experiments, $2 \times 10^{4} \mathrm{CD} 20+$ human B-cells/well were cultured in the growth medium supplemented with $10 \%$ of serum (either FCS or iHS or HS) with or without rituximab. Survival in the control group (cell growth medium and FCS) was taken as $100 \%$.

\section{Flow cytometry}

Staining for the presence of $C D 20$ antigen. The CD20+ human B-cells $\left(1 \times 10^{6}\right)$ were spun down at $1000 \mathrm{rpm} / \mathrm{min}$. The sediment was carefully resuspended in the residual fluid and $100 \mu \mathrm{l}$ of culture medium was added (MeM with 2\% inactivated FCS). The cells were stained with $10 \mu \mathrm{l}$ of CD20 monoclonal antibodies conjugated with PE-Cy5 (BD Biosciences Pharmingen, San Diego, CA, USA) for 30 minutes at room temperature in the dark and then washed 3 times using MeM and resuspended in Facsflow solution (BD Biosciences, San Diego, CA, USA).

Apoptosis - Annexin test. For the detection of apoptosis, Annexin $\mathrm{V}$ test was used (BD Pharmingen, San Diego, CA, USA). The CD20+ human B-cells were resuspended in $100 \mu \mathrm{l}$ of binding buffer and stained with 5 $\mu \mathrm{l}$ Annexin V-FITC reagent and $10 \mu \mathrm{l}$ of propidium iodide (Molecular probes, Eugene, Oregon, USA) for 15 minutes at room temperature in the dark. After incubation, 400 $\mu \mathrm{l}$ of binding buffer was added to each tube. Measurements were performed within one hour.

Adjustment of the flow cytometer. Flow cytometer (FACSort, Becton Dickinson, San Jose, CA, USA) was adjusted for the optimal acquisition of lymphocytes. In the dot plot screen $\{\mathrm{FSC}=(\mathrm{X})$ toward the SSC $=(\mathrm{Y})\}$, the lymphocyte-like population was gated. In the histogram screen, PE-Cy peak was selected and transferred into the dot plot screen. The percentage of live, apoptotic and dead CD20+ human B-cells was calculated using the quadrant statistic tool.

\section{Statistical analysis}

The in vitro experiments were set up in triplicates and the results were expressed as means $\pm \mathrm{SD}$. The results were analyzed for statistical significance of difference between the groups using the unpaired two 
tail Student's $t$ test. The $p$ level $<0.05$ was considered as statistically significant.

\section{Patients}

In the clinical part of the research, 20 patients with aggressive CD20 positive NHL were treated with R-CHOP at the Department of Medical Oncology of the Institute of Oncology Ljubljana, Slovenia, from October 2003 to February 2005. The research included 6 male patients with the median age of 66.5 years (range 44 to 74 ) and 14 female patients with the median age of 63.5 years (range 37 to 78). Seventeen patients were diagnosed with diffuse large B-cell lymphoma and 3 patients had follicular lymphoma grade 3 . The R-CHOP treatment was first-line treatment for newly diagnosed lymphomas and all patients included in the study had advanced lymphomas (stages III and IV according to Ann Arbor staging).

Rituximab (kindly donated by Roche) was applied at standard doses $\left(375 \mathrm{mg} / \mathrm{m}^{2}\right)$ intravenously on day 1 , followed by CHOP chemotherapy (cyclophosphamide $750 \mathrm{mg}$ / $\mathrm{m}^{2}$, doxorubicyn $50 \mathrm{mg} / \mathrm{m}^{2}$ and vincristine $2 \mathrm{mg}$, unless adjusted to the patient's performance status or previous toxicity). Premedication with methylprednisolone, paracetamole and clemastine was given prior to every rituximab application. The cycles of chemo-immunotherapy were repeated every three weeks. The majority of patients received 8 cycles of such treatment, one patient died of complications after the first cycle, and in one patient, the treatment was terminated due to disease progression after the sixth cycle. Eleven patients received intrathecal chemotherapy in order to prevent the spreading of lymphoma into CNS, and in two patients, middle doses of methotrexate were added to R-CHOP due to concomitant CNS localization of lymphoma.
Treatment response was evaluated after the $4^{\text {th }}$ and after the $8^{\text {th }}$ cycle of treatment according to Cheson's criteria. ${ }^{6}$

\section{Results}

\section{Cell growth inhibition by rituximab}

In order to determine the in vitro effect of rituximab on CD20+ B lymphocytes, Raji and SU-DHL-4 cell lines were grown in the growth medium supplemented with rituxi$\mathrm{mab}$ and either $10 \%$ of inactivated FCS (FCS) or $10 \%$ of inactivated human serum (iHS) or $10 \%$ of intact human serum (HS). The viability of cells after $24 \mathrm{~h}, 48 \mathrm{~h}$ and $72 \mathrm{~h}$ was determined using the cell proliferation assay.

The growth of both Raji and SU-DHL-4 cells was inhibited by rituximab in the presence of HS. In Raji cells, the inhibition was observed at all time points - at $24 \mathrm{~h}, 48 \mathrm{~h}$ and $72 \mathrm{~h}$. The difference was statistically significant at all time points when compared to the cell growth of cells cultured only with iHS or HS ( $p$ values were in the range of 0,045 to 0,003 ) (Figure 1A). When comparing with the growth of the cells cultured with rituximab and FCS or iHS, a statistically significant growth reduction of the cells cultured with rituximab in the presence of HS was obtained only after $72 \mathrm{~h}$. The $p$ values were $0.026(\mathrm{R}+\mathrm{iHS} / \mathrm{R}+\mathrm{HS})$ and $0.003(\mathrm{R}+\mathrm{FCS} /$ $\mathrm{R}+\mathrm{HS})$, respectively (Figure $1 \mathrm{~A})$.

In SU-DHL-4 cells, a statistically significant growth reduction was obtained for the cells cultivated with rituximab in the presence of HS compared to all other groups at $48 \mathrm{~h}$ and $72 \mathrm{~h}$. The $p$ values were in the range of 0.012 to 0.001 . After $24 \mathrm{~h}$, the only statistically significant growth reduction was observed when we compared the growth of the cells cultured with rituximab in the presence of HS with the growth of the cells cultured only with iHS $(p=0.013)$ (Figure 1B). 
Determination of mechanisms of rituximab action

In order to confirm the mechanisms by which rituximab induces killing of $\mathrm{CD} 20+\mathrm{B}$ lymphocytes, Raji cells were incubated with rituximab for $4 \mathrm{~h}, 24 \mathrm{~h}$, and $48 \mathrm{~h}$ in the presence of HS (20\%) or hPBMC (ratio 1:1) and the proportions of alive, apoptotic, or dead cells were determined by flow cytometry.

There was no difference in the proportions of alive, dead or apoptotic cells between the cells that were grown in the medium supplemented with FCS or iHS and the cells grown in the same medium, but with added rituximab (data not shown). However, the ratio of apoptotic cells was substantially increased in those cells that had been co-incubated with rituximab in the presence of $20 \%$ HS. Comparing these cells to the cells cultured only with HS, a significantly increased proportion of apoptotic cells was obtained after $24 \mathrm{~h}$ and after
$48 \mathrm{~h}$. The relating $p$ values were 0.001 after $24 \mathrm{~h}$ and 0.003 after $48 \mathrm{~h}$. Similar results were obtained when the ratios of apoptotic cells among the cells incubated with rituximab in the presence of $20 \%$ HS and among the cells cultured only with FCS were compared. The relating $p$ values were 0.017 after $24 \mathrm{~h}$ and 0.009 after $48 \mathrm{~h}$ (Figure 2).

In our study, hPBMC (isolated from the same blood sample as human serum) did not affect significantly the number of apoptotic cells either when used alone or in combination with rituximab. There was also no statistically significant difference in the number of apoptotic cells when CD20+ B lymphocytes were co-incubated with rituximab and hPBMC in the presence of FCS or when co-incubated only with hPBMC in the presence of FCS. Significant difference was not observed even when the results obtained with rituximab and hPBMC were compared to other groups.
A.

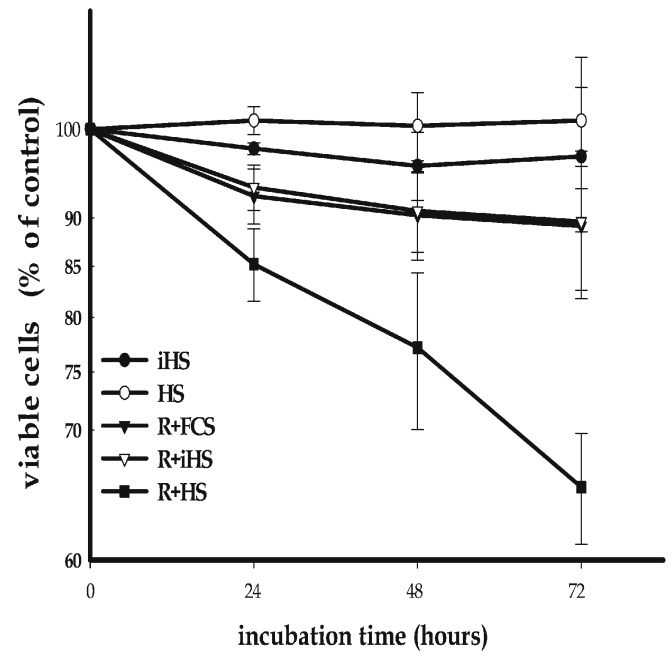

B.

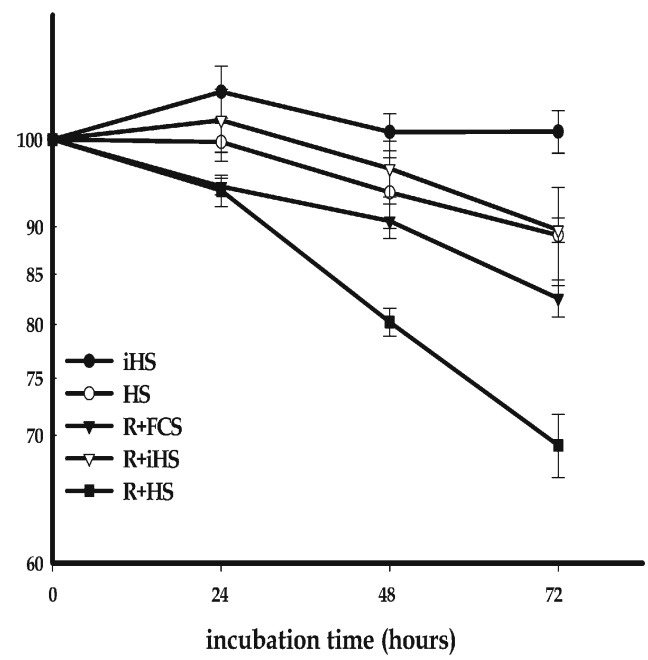

Figure 1. Growth of lymphoma CD20+ Raji (panel A.) and SU-DHL-4 cells (panel B.) in the presence of $20 \mu \mathrm{g} / \mathrm{ml}$ of rituximab. The plots represent the $\mathrm{AM} \pm \mathrm{SD}$ of three independent experiments. The proportion of viable cells in experimental groups was calculated as \% of the control (cells grown in medium supplemented with FCS).

iHS - cells grown in $10 \%$ of inactivated human serum, HS - cells grown in $10 \%$ of intact human serum, R+FCS - cells grown with rituximab in $10 \%$ of inactivated fetal calf serum, $\mathbf{R}+\mathbf{i H S}$ - cells grown with rituximab in $10 \%$ of inactivated human serum, $\mathbf{R}+\mathbf{H S}$ - cells grown with rituximab in $10 \%$ of intact human serum. 


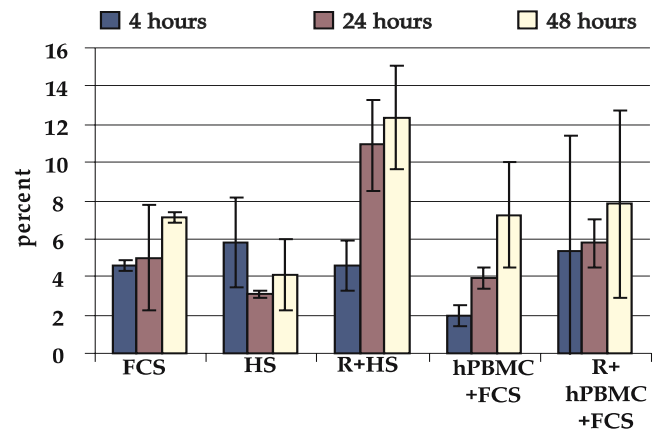

Figure 2. Proportion of apoptotic cells among Raji cells incubated with rituximab for $4 \mathrm{~h}, 24 \mathrm{~h}$, and $48 \mathrm{~h}$ in the presence of HS (20\%) or hPBMC (ratio 1:1) determined by flow cytometry. The plots represent the $\mathrm{AM} \pm \mathrm{SD}$ of three independent experiments. FCS - cells grown in $2 \%$ of inactivated fetal calf serum, HS - cells grown in $20 \%$ of intact human serum, $\mathbf{R}+\mathbf{H S}$ - cells grown with rituximab in $20 \%$ of intact human serum, hPBMC+FCS - cells grown in $2 \%$ of FCS with fresh hPBMC, $\mathbf{R}+\mathbf{h P B M C + F C S}$ - cells grown with rituximab in $2 \%$ of FCS and in the presence of fresh hPBMC.

\section{Saturation of $C D 20$ receptors by rituximab}

Binding of rituximab to cell receptors was followed by flow cytometric determination of free CD20 receptors after $4 \mathrm{~h}, 24 \mathrm{~h}$ and $48 \mathrm{~h}$ when $1 \times 10^{6}$ Raji cells were co-incubated with $20 \mu \mathrm{g} / \mathrm{ml}$ of rituximab in the presence of FCS. As the control, the same number of Raji cells without rituximab was used. Four hours following the addition of rituximab, only $30.7 \%$ of CD20 receptors remained free. However, after $24 \mathrm{~h}$ and $48 \mathrm{~h}$ the proportion of free CD20 receptors increased to $75.6 \%$ and $66.0 \%$, respectively (Figure 3 ).

\section{Clinical results}

According to Ann Arbor staging system, 3 patients had stage III.A disease, 3 patients III.A.E, 1 patient III.B, 1 patient IV.A, 5 patients IV.A.E, and 7 patients IV.B.E disease. Extranodal localizations of lymphoma were found in as much as 15 patients (and in some patients they were multiple). Five

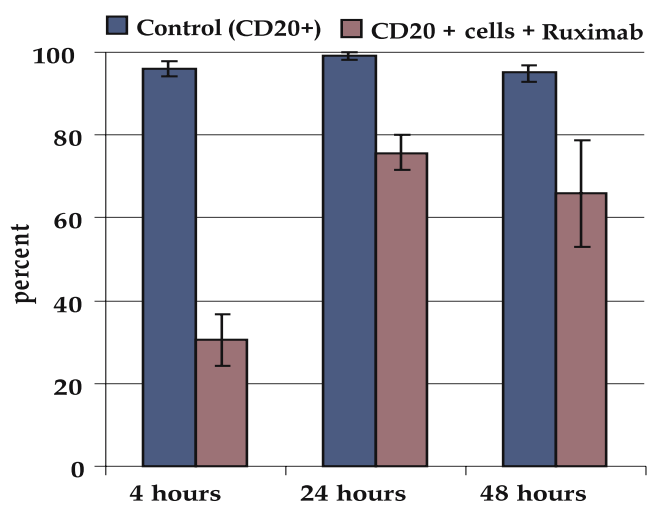

Figure 3. Saturation of CD20 receptors on Raji cells by rituximab after different periods of time. Raji cells $\left(1 \times 10^{6}\right)$ were co-incubated with $20 \mu \mathrm{g} / \mathrm{ml}$ of rituximab in the presence of FCS. As the control, the same number of Raji cells without rituximab was used. The plots represent the $\mathrm{AM} \pm \mathrm{SD}$ of three independent experiments.

patients had lymphomatous infiltration of the stomach/intestine, 4 had skeletal involvement, 2 CNS involvement, 4 patients lung and 2 patients pleural infiltration, 2 patients pancreatic and 1 renal infiltration, in 4 patients, there was soft tissue involvement (skin or muscles), and in 4 patients, infiltrates were detected in the ORL region (tonsils, maxillary sinus, salivary glands).

The international prognostic index was low intermediate (2) in 5 patients, high intermediate (3) in 8 patients, and high (4 or $5)$ in 7 patients. The serum LDH concentrations were also elevated in 15 patients, with the highest concentration reaching $29 \mu \mathrm{kat} / \mathrm{l}$ (i.e. approximately seven-times the upper normal limit).

During the treatment, some grade 3 or 4 toxicities developed in 15 patients and only the remaining 5 had practically no or only minor side effects. Among the most common side effects were neutropenia with or without fever (in 14 patients) and serious infection/septicemia (in 6 patients), all of which resulted in the necessity for drug dosage reductions in 7 patients. 
Table 1. Treatment response to R-CHOP in patients with aggressive CD20 Non-Hodgkin's lymphomas.

\begin{tabular}{llll}
\hline $\begin{array}{l}\text { Treatment } \\
\text { response }\end{array}$ & $\begin{array}{l}\text { After } \mathbf{4}^{\text {th }} \\
\text { cycle }\end{array}$ & $\begin{array}{l}\text { After } 8^{\text {th }} \\
\text { cycle }\end{array}$ & $\begin{array}{l}\text { After } \\
\text { RT* }\end{array}$ \\
\hline PR & $8(40 \%)$ & $4(20 \%)$ & \\
CR & $10(50 \%)$ & $14(70 \%)$ & $18(90 \%)$ \\
SD & $2 * *$ & & \\
PD & & $1 * * *$ & \\
\hline
\end{tabular}

* - just the 4 patients with PR after the $8^{\text {th }}$ cycle were irradiated

** - in 1 patient there was stable disease (IPI 4), another one died 2 days after the first cycle (IPI 3 ) *** - PD after the $6^{\text {th }}$ cycle (IPI 4 )

After the $4^{\text {th }}$ cycle of treatment, partial response (PR) was observed in 8 patients, complete response (CR) in 10 patients, and stable disease (SD) in 1 patient. One patient died 2 days after the first cycle in septic shock most probably due to necrotizing pancreatitis. This patient had a lymphomatous infiltration of the pancreas confirmed prior to the introduction of treatment.

At the end of systemic treatment, 4 of 8 patients with prior partial remission achieved complete remission, while in the other 4 , the final outcome was partial remission. These 4 patients continued their treatment with irradiation of the residual lymphoma masses and eventually achieved complete remission. The patient with stable disease following the $4^{\text {th }}$ cycle progressed (PD) after another two cycles of treatment and was further on treated with another chemotherapy schedule. This patient died of progressive lymphoma 4 months after the last R-CHOP cycle (Table 1).

The complete response still lasts in 16 patients $(80 \%)$ with the median observation period of 18.5 months (range 12 to 15.5 months) and the median response duration has not been reached yet (Figure 4). On the other hand, two patients progressed (both IPI 3), one 5.5 and the other 9.5 months after the end of treatment. These two patients were subsequently treated with different

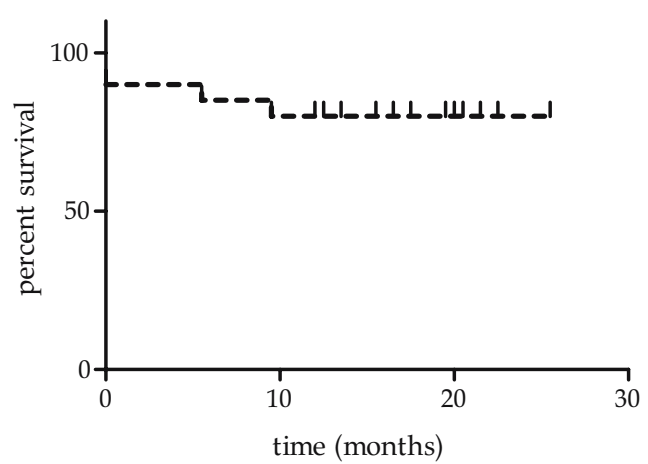

Figure 4. Disease free survival in 20 high-risk patients with aggressive CD 20 lymphoma after treatment with R-CHOP.

chemotherapy schedules and are still alive - one of them in the second remission and the other one still under treatment.

\section{Discussion}

Clinical application of rituximab in combination with chemotherapy has significantly improved the treatment outcome in the patients with indolent and aggressive non-Hodgkin's lymphoma (NHL). ${ }^{4}$ Still, the exact in vivo mechanisms of action of rituximab are not fully understood, although antibody-dependent cell-mediated cytotoxicity (ADCC), complement-dependent cytotoxicity (CDC), and apoptosis have been suggested. ${ }^{7}$ This study, was aimed to confirm the presumed major mechanisms of action of rituximab and concomitantly to assess the effectiveness of first-line chemoimmunotherapy with R-CHOP in the highrisk patients with aggressive CD20 NHL.

The results of in vitro experiments on Raji and SU-DHL-4 cells provided evidence for a significant effect of rituximab on the cell growth starting more than $24 \mathrm{~h}$ after the exposure to the drug, but only when the cells were grown in the presence of intact HS. Culturing of these cells with rituximab and inactivated FCS or HS did not result in 
a significant growth reduction alluding to the possibility that, in in vitro conditions, rituximab by itself was not sufficient to affect critically the tested cells. The observation was unexpected since certain previous studies validated this very mechanism of action of rituximab. ${ }^{7-10} \mathrm{~A}$ gene expression analysis revealed that the binding of rituximab to CD20 receptors induces in vitro activation of genes known to be involved in the cell growth control and apoptosis. In the study of Jazirehi et al., evidence was provided that rituximab treatment of B-cell lines Ramos and Daudi inhibits the constitutive NF- $\mathrm{BB}$ signaling pathway thereby down-regulating the $\mathrm{Bcl}-\mathrm{x}_{\mathrm{L}}$ expression. In this way, the antibody directly affects cells through CD20 receptors diminishing their proliferative activity and, even more, rituximab also sensitizes tumor cells to the cytotoxic activity of certain drugs. ${ }^{10}$ Due to the observed discrepancy between the stated results and our results, we expanded the in vitro experimentation to flow cytometry. The effects of rituximab were thus followed by the determination of proportions of apoptotic, dead and alive cells among the differently treated cells. Surprisingly, also the results obtained from the flow cytometric analysis confirmed our previous observations. There was a statistically significant reduction in the number of live cells (for the groups $\mathrm{HS} / \mathrm{R}+\mathrm{HS}$ after $24 \mathrm{~h}$ and $48 \mathrm{~h}$ the $p$ values were 0.005 and 0.044 , respectively) and a statistically significant increase of apoptotic cells, but only among the cells treated with rituximab in the presence of intact $\mathrm{HS}$ (for the groups HS/R+HS after $24 \mathrm{~h}$ and $48 \mathrm{~h}$ the $p$ values were 0,001 and 0,003 , respectively). The anticipated increase in the number of apoptotic cells among cells treated with rituximab in the presence of iHS or FCS was not observed. Since the degree of apoptosis and growth conditions in the studies evaluating this mechanism varied from study to study ${ }^{7}$, it is quite possible that the model used in our study was not sensitive enough to detect the changes as direct consequences of the monoclonal binding to CD20 receptors. Therefore, the next step in our investigation was the determination of the extent of rituximab binding and saturation of CD20 receptors on the target cells. Using $20 \mu \mathrm{g}$ of rituximab per one $\mathrm{ml}$ of cell growth medium, the saturation of CD20 receptors was the highest $4 \mathrm{~h}$ after the beginning of treatment (almost 70\%). In the next hours, the saturation rapidly decreased indicating that the initial concentration of rituximab was probably too low - which may actually be one of the reasons why direct apoptosis due to cross-linking of CD20 has not been observed. Additionally, in some studies of normal and malignant human $B$ cells in vitro, B-cell depletion was observed predominately with rituximab in the presence of mononuclear cells, but not in the presence of a complement, suggesting the importance of cell-mediated mechanisms (ADCC).${ }^{11}$ In other studies, however, both the activation of ADCC and complementmediated lysis have been demonstrated in vitro and in vivo after the treatment with rituximab. ${ }^{12-14}$ Our in vitro testing failed to demonstrate any significant difference in the number of alive, apoptotic or dead cells between the cells co-incubated only with hPBMC or with hPBMC in combination with rituximab. Most probably, the reason for this is an inadequate ratio between hPBMC and CD20+ B-cells. This ratio was 1:1 in our study, meaning that the ratio of the cells capable of mediating the cytotoxic response, i.e. NK cells and monocytes, was at the best 1:1 or lower, making it unlikely that cytotoxicity had any significant impact on the proportion of dead cells.

When commenting the clinical data, the treatment results in the poor-risk patients with aggressive NHL were disappointing in the pre-rituximab era as reported by Shipp et al in $1993 .{ }^{15}$ For example, complete remis- 
sion was achieved in only $44 \%$ of patients with high IPI and in 55\% of patients with high intermediate IPI as compared to $87 \%$ of patients with low IPI. In our research, predominately the patients with high intermediate ( 8 patients) and high IPI (7 patients) were included and not one single patient had low IPI. Therefore, the fact that as much as $90 \%$ of these patients finally achieved complete remission (14 patients after chemoimmunotherapy and 4 patients after additional radiotherapy) definitely speaks for superior activity of R-CHOP compared to chemotherapy alone in the high-risk patients (in spite of the small number of the patients included in our study).

Also the rate of disease free survival was much higher in our study $(80 \%$ of the patients) compared to the data of Shipp et al. ${ }^{15}$ who reported 58\% and 59\% 2-year relapsefree survival for high and high intermediate IPI groups, respectively, even though our median observation period was only 18.5 months.

Obviously the addition of rituximab to the treatment protocol for the poor-risk patients with aggressive NHL improved the previous treatment results achieved only with chemotherapy ( \pm radiation treatment). This improvement could be the reflection of an increased sensitivity to chemotherapeutic agents induced by rituximab which is in accordance with the in vitro results of the two lately published studies. ${ }^{10,11}$

\section{Conclusions}

The results of our study indicate that the complement-dependent cytotoxicity (CDC) is an important mechanism of rituximab action in vitro since it seems to be triggered easily even if the absolute saturation of CD20 receptors is not reached. To achieve direct apoptosis, higher concentration of rituximab $(>20 \mu \mathrm{g} / \mathrm{ml})$ should be used while for an effective ADCC, the ratio of cytotoxic lymphocytes to tumor cells should be increased (to more than $>1: 1$ ). From the clinician's point of view, the addition of rituximab to $\mathrm{CHOP}$ in the high-risk patients with aggressive CD20 NHL is obligatory if the goal of the treatment is to cure the patient. Accordingly, it is no exaggeration to state that rituximab changes the prognosis of poor-risk patients with aggressive CD20 NHL.

\section{Acknowledgements}

This research was supported by Roche (donation of rituximab for the in vitro and clinical part of the study) and Slovenian Ministry of Science (grant J3-6363).

\section{References}

1. Lucas BJ, Horning SJ. Monoclonal antibodies have finally arrived. In: Cavalli F, Armitage JO, Longo DL, editors. Annual of Lymphoid Malignancies. London: Martin Dunitz Ltd; 2001. p. 153-67.

2. Jazirehi AR, Bonavida B. Cellular and molecular signal transduction pathways modulated by rituximab (rituxan, anti-CD20 mAb) in non-Hodgkin's lymphoma: implications in chemosensitization and therapeutic intervention. Oncogene 2005; 24: 2121-43.

3. Maloney DG, Liles TM, Czerwinski DK, Waldichuk C, Rosenberg J, Grillo-Lopez A, et al. Phase I clinical trial using escalating single-dose infusion of chimeric anti-CD20 monoclonal antibody (IDECC2B8) in patients with recurrent B-cell lymphoma. Blood 1994; 84: 2457-66.

4. Boye J, Elter T, Engert A. An overview of the current clinical use of the anti-CD20 monoclonal antibody rituximab. Ann of Oncol 2003; 14: 520-35.

5. Maloney DG. Mechanisms of action of rituximab. Anticancer Drugs 2001; 12 Suppl 2: S1-S4.

6. Cheson BD, Horning SJ, Coiffier B, Shipp MA, Fisher RI, Connors JM, et al. Report of an international workshop to standardize response criteria for Non-Hodgkin's lymphomas. J Clin Oncol 1999; 17: $1244-53$. 
7. Smith MR. Rituximab (monoclonal anti-CD20 antibody): mechanisms of action and resistance. Oncogene 2003; 22: 7359-68.

8. Cittera E, Onofri C, D'Apolito M, Cartron G, Cazzaniga G, Zelante L, et al. Rituximab induces different but overlapping sets of genes in human B-lymphoma cell lines. Cancer Immunol Immunother 2005; 54: 273-86.

9. Smith MR, Joshi I, Jin F, Obasaju C. Enhanced efficacy of gemcitabine in combination with antiCD20 monoclonal antibody against CD20+ nonHodgkin's lymphoma cell lines in vitro and in scid mice. BMC Cancer 2005; 5: 103.

10. Jazirehi AR, Huerta-Yepez S, Cheng G, Bonavida B. Rituximab (chimeric anti-CD20 monoclonal antibody) inhibits the constitutive nuclear factor- $\mathrm{\kappa} B$ signaling pathway in non-Hodgkin's lymphoma B-cell lines: role in sensitization to chemotherapeutic drug-induced apoptosis. Cancer Res 2005; 65: 264-76.

11. Clynes RA, Towers TL, Presta LG, Ravetch JV. Inhibitory Fc receptors modulate in vivo cytoxicity against tumor targets. Nat Med 2000; 6: 443-6.

12. Reff ME, Carner K, Chambers KS, Chinn PC, Leonard JE, Raab R, et al. Depletion of B cells in vivo by a chimeric mouse human monoclonal antibody to CD20. Blood 1994; 83: 435-45.

13. Golay J, Zaffaroni L, Vaccari T, Lazzari M, Borleri GM, Bernasconi S, et al. Biologic response of B lymphoma cells to anti-CD20 monoclonal antibody rituximab in vitro: CD55 and CD59 regulate complement mediated lysis. Blood 2000; 95: 39008.

14. Winkler U, Jensen M, Manzke O, Schulz H, Diehl $\mathrm{V}$, Engert A. Cytokine-release syndrome in patients with B-cell chronic lymphocytic leukemia and high lymphocyte counts after treatment with an anti-CD20 monoclonal antibody (rituximab, IDEC-C2B8). Blood 1999; 94: 2217-24.

15. Shipp MA, Harrington DP, Anderson JR, Armitage JO, Bonadonna G, Brittinger G, et al. A predictive model for aggressive non-Hodgkin's lymphoma. $N$ Engl J Med 1993; 329: 987-94. 